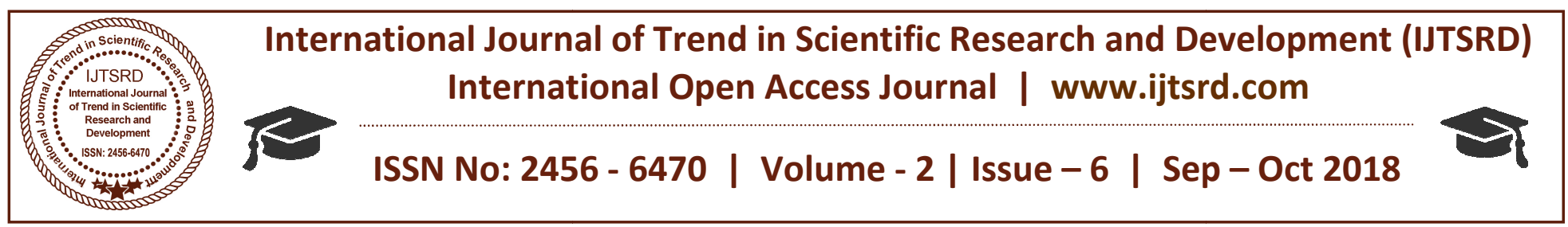

\title{
Multi Model Biometrics Data Retrieval Through: Big-Data
}

\author{
Mr. Manish Vala ${ }^{1}$, Kajal Patel ${ }^{2}$, Harsh Lad ${ }^{2}$ \\ ${ }^{1}$ Assistant Professor, ${ }^{2}$ Student \\ Babumadhav Institute of Information Technology Bardoli, Gujarat, India
}

\section{ABSTRACT}

This study is devoted to Big Data problems in biometric technologies. Before the traditional database were not capable to handle the large amount of data, so we highlight the concept of using Big Data technology in solution of a wide range of issues faced by the national security, law enforcement system and defence authorities. The study provides information about Big Data in the field of biometric technology.

Keyword: Biometrics, Big Data, Recognition, Hadoop.

\section{INTRODUCTION}

\section{$>$ Big Data}

Big data means really a big data; it is a collection of large datasets that cannot be processed using traditional computing techniques or it is set of data that are voluminous and complex data. Big Data challenges include capturing data, data storage, data analysis, search, sharing, transfer, visualization, querying, updating, and information privacy

The main purpose of Big Data is to improve efficiency for using the large volume of data. If the Big Data is used in proper manner than organizations can pursue new business model and can achieve competitive advantages.

It includes huge volume, high velocity, and extensible variety of data.

The data in it can be of three types:

1. Structured data: It can be Relational Data.

2. Semi Structured data: They are in form of XML data.

3. Unstructured data: It consists of Word, PDF, Text, Media files.

\section{$>$ Characteristics of Big Data}

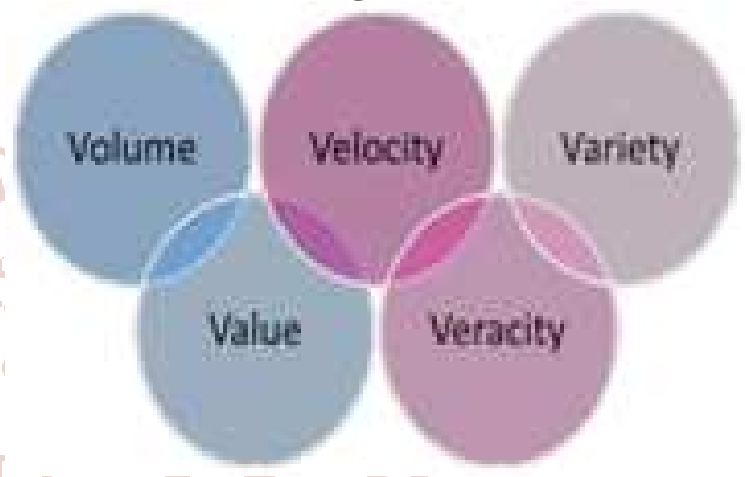

Figure1. Characteristics of Big Data [13]

\section{Volume:}

When talking about the Volume, it refers to the insanely large amount of data or quantity of data.

\section{Variety:}

It refers to the different data types, degree of structured, semi-structure and unstructured data which doesn't fit easily into database application.

3. Velocity:

It refers to the speed of data how fast the data are generated every second. Example: Google, Facebook.

\section{Veracity:}

It refers to the trustworthiness of the data in terms of accuracy.

\section{Value:}

It means having large amount of data is good, but it is useless if it can't be turned into values.

\section{Advantages of Big Data}

1. Using the information kept in the social network the marketing agencies are learning about the response for the campaigns, promotions, and other advertising medium. Ex:-Instagram.

2. Companies are allowed to store more transactional data in digital format.

3. Using the data regarding the pervious medical history patients, hospital are providing better and quick service. 
4. Some grate benefits of Big Data are cost reduction, Real time website customization, swift - better decision making.

\section{$>$ Biometric Recognition}

It is a technology used to identify, analyze and measure an individual's physical and behavioural characteristics. Those data are captured from the human body and are compared to the data that are stored in set of database.

The common biometric authentication techniques used is Finger prints biometric authentication. Some other techniques which can be used are Face recognition, Iris Scan, voice Recognition. Today biometrics system have been implemented in various commercial, civilian and forensic application as a means of establishing identity.

Biometrics is used for authenticating and authorizing a person. Authentication of a person through biometric is highly challenging. It is used in forensics, secured access, prison security, medical, and robotics areas financial services, In ecommerce, telecommunication, government, traffic and many more. New technology or technique can be used is ear recognition of individuals.

Biometrics authentication has two types of Model for Authentication Process:

\section{Unimodal}

Unimodal biometrics refers to the use of only one biometrics modalities in an identification system.

Example: Fingerprint recognition

\section{Multimodal}

Multimodal biometrics refers to the use of a combination of two or more biometrics modalities in an identification system

Example: Face recognition and Iris scan.
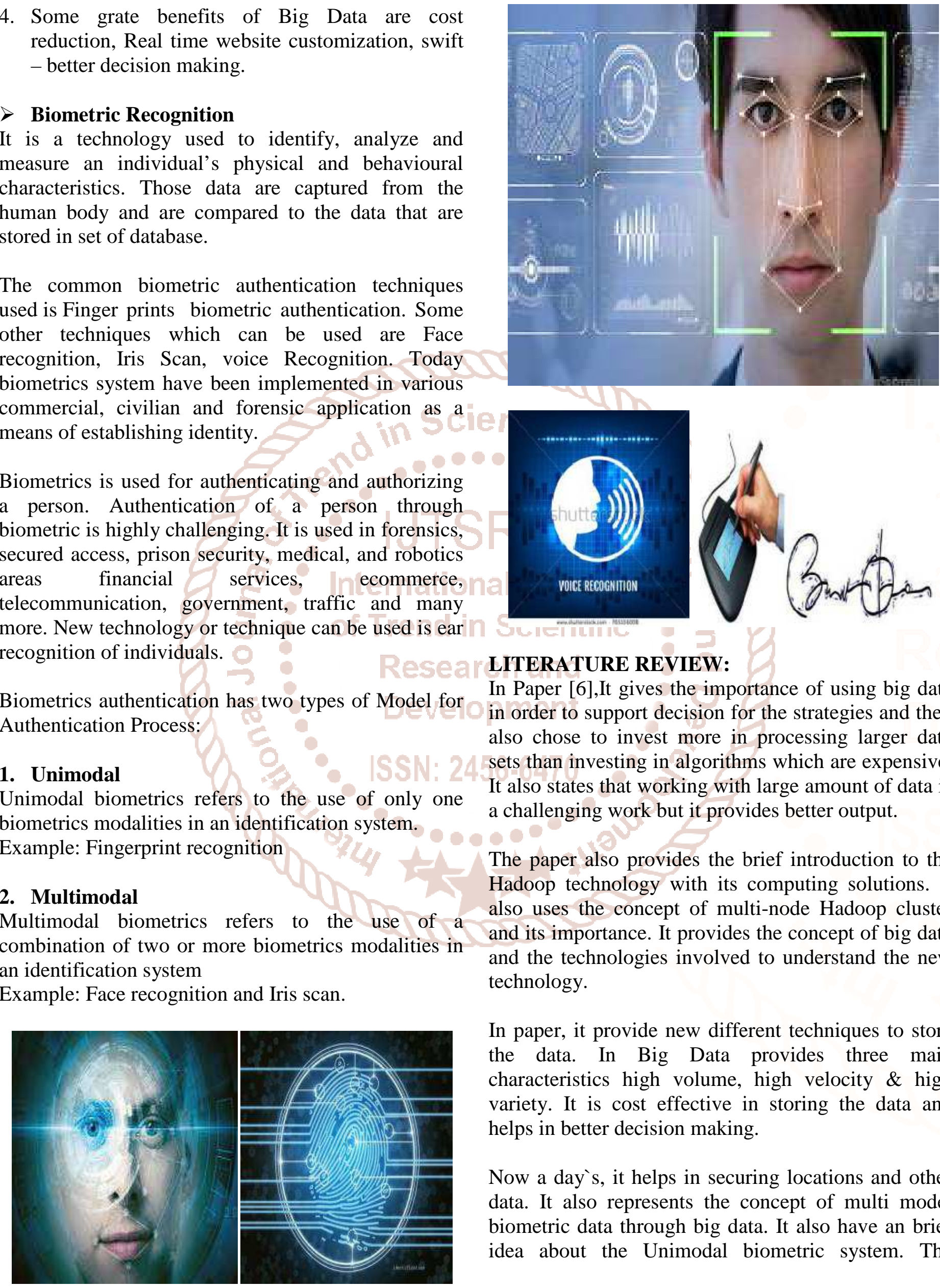

LITERATURE REVIEW:

In Paper [6],It gives the importance of using big data in order to support decision for the strategies and they also chose to invest more in processing larger data sets than investing in algorithms which are expensive. It also states that working with large amount of data is a challenging work but it provides better output.

The paper also provides the brief introduction to the Hadoop technology with its computing solutions. It also uses the concept of multi-node Hadoop cluster and its importance. It provides the concept of big data and the technologies involved to understand the new technology.

In paper, it provide new different techniques to store the data. In Big Data provides three main characteristics high volume, high velocity \& high variety. It is cost effective in storing the data and helps in better decision making.

Now a day`s, it helps in securing locations and other data. It also represents the concept of multi model biometric data through big data. It also have an brief idea about the Unimodal biometric system. The 
biometrics devices typically used are hand geometry reader and fingerprint authentication.

In Paper[8], discussed about the big data and big data challenges in biometrics. It highlights the use of big data technologies. It also provides characteristics of the big data and types of data which can be generatedstructured, semi structured unstructured data. It provides the information about the biometrics and problems that are occurred in biometric technologies.

Brief discussion about the cloud technologies regarding how the cloud technology can be used to recognize the person's characteristics. They are working on developing advance big data capabilities to prevent from terrorism and criminal investigation.

In Paper [9], Discussed about the Unimodal and multi-modal biometric system. Multi-modal provides the reasons for reducing the rates. Levels of Fusion used in multimodal biometric system. It also provides the comparison between the Unimodal and multimodal and limitations of Unimodal can be overcome by the multimodal.

In paper [2], The discussion is done about the ear recognition system. It is the currently used technology in biometric identification of human and it is quite complex task. It also provides the brief information about the model used for biometric authenticationUnimodal and multimodal.

It also provides discussion about the existing techniques of human Authentication and identification - fingerprints (overview of fingerprint recognition with its operation, limitation and area of application and what instruments are required to identify the fingerprints.) same for face recognition and Iris scan. Main it provides how the ear recognition is done and the usefulness of ear based biometric identification system.

In Paper [10], Multimode biometrics is suitable for mobile, PDAs, smart phones. They are portable subject to loss and theft. The use of enhanced security mechanism as biometric authentication. The use of teeth image and voice authentication. It is fact that the military deploys largest use of mobile devices of which are embedded system with tactics and military intelligence. Authentication to such by teeth image and voice, it will become difficult to the security of such device in the event of its loss or misplace.
In Paper [11],Discussion about multimodal biometrics application and research area. It is used to combine two or more different sources of person sensed by different sensors. It include border management, interface for criminal and civil applications, and first responder verification. System can be improved by enhancing matching algorithms, integration of multiple sensor, and analysis of the scalability of biometrics system.

Signature recognition requires little space to store the data. A disadvantage for signature recognition is that individuals who do not sign their names in a consistent manner may have difficulty enrolling and verifying in signature verification. Iris scan is believed to be highly stable over lifetime and uses an infrared imager to illuminate the eye and capture a very high-resolution image. Iris scan is difficult to capture for some individuals. In voice recognition sound sensations of a person is measured and compared to an existing dataset. Voice recognition Difficult to control sensor and channel variances that significantly impact.

\section{PROPOSED MODEL}

The multi model biometric data handling through big data framework is proposed. The multi model biometric framework first reads biometric (image, video, sound, etc.) data from user. Once the data is collected from the user the data is been entered into application framework. It identifies the biometric type and converts those data into binary form, same procedure is followed for other biometric data. The converted data are been merged into single biometric detail by using the algorithm. The binary data are now encrypted using the encryption algorithm.

The authentication process, done when the user comes for biometric authentication, the multi model biometric framework reads the data of user than identify the type of biometric data and converts the data into binary data. Binary data is encrypted and those encrypted data is compared to the biometric data. If the compared data is matched with the data in database than it retrieves the binary data. After extracting binary data from big data Hadoop framework API, data will be decrypted and convert into original data form and user will get the real time authentication though multi model biometric framework. 


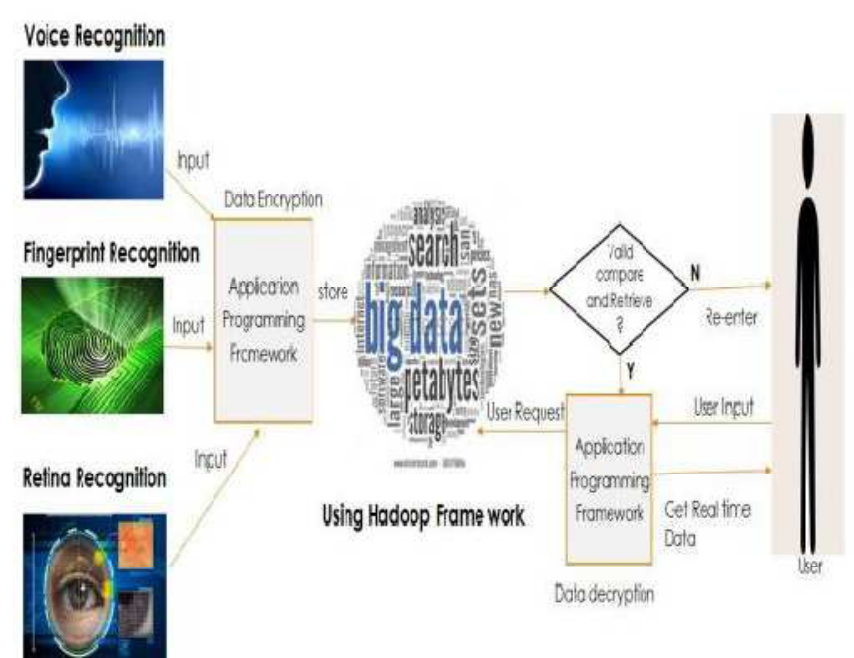

Figure 7 Proposed Model of Hadoop [20]

\subsection{Map - Reduce Algorithm:}

It is a distributed data processing algorithm, useful for processing huge amount of data in cluster environment.

This algorithm uses the concept of divide and conquer algorithm to process large amount of data.

The input is divided into smaller and manageable subtasks to execute them parallel.

This algorithm uses three main steps:

1. Map Function

2. Shuffle Function

3. Reduce Function

\section{PROPOSED ALGORITHM:}

\section{> K-means clustering algorithm:}

This algorithm is used for creating and analyzing the clusters. ' $\mathrm{N}$ ' number of data points are divided into ' $\mathrm{k}$ ' cluster. Results generated from the algorithm is dependent on choosing the initial cluster centroid.

The main feature, if data is arranged into a matrix, with row representing data points and column representing attributes of each data points, than matrix might not have defined values at many places.

\section{Algorithm:}

All data points have maximum $\mathrm{M}$ dimension which can be represented as row of $\mathrm{M}$ values $(\mathrm{v} 1, \mathrm{v} 2 \ldots . .$. $\mathrm{vM})$.Select any $\mathrm{m}$ dimension from the data points. Arrange them in descending order of priority as, (d1, $\mathrm{d} 2 \ldots, \mathrm{dm})$. The first dimension is known as primary dimension and others are called secondary dimension. Number of cluster is pre-decided-' $\mathrm{k}$ ' which is an input to the algorithm.

Step 1: the variation of each dimension is considered as, $(\max -\min ) / \mathrm{k}, \quad 1<=\mathrm{i}<=\mathrm{m}$. where max is the maximum value of $\mathrm{i}$ dimension and the min is the minimum value of $i$ dimension.

Step 2: Initial cluster are formed using following conditions for data point, if $\min 1+\mathrm{j} * \$ 1<=$ val1 $<$ mi $1+(\mathrm{j}+1) * \$ 1$, the data point belongs to cluster $\mathrm{j}$.

Step 3: Centroid of each cluster is computed means of all cluster points.

Step 4: Every secondary dimension, $2 \leq j \leq \mathrm{m}$, repeat the same.

\section{Algorithm:}

Map function: In this step, it takes input tasks and divide the task into smaller sub-tasks. It follows two sub-steps:

1. Splitting: takes input and divide into smaller subdatasets.

2. Mapping: takes smaller sub-datasets and perform action on each sub-datasets.

Shuffle function: In this step, also known as combine phase. It performs two sub-steps:

1. Merging: combines all key-value pairs which have same key.

2. Sorting: takes the input from merging step and sort all key-value pair by using keys.

Reduce function: In this step, it takes the list of sorted key-value pairs from shuffle function and perform reduce operation.

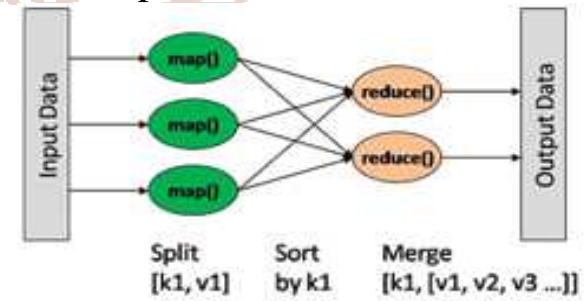

Figure8. Architecture of Map - Reduce [18]

\section{Conclusion and Future work:}

The literature survey done in this paper provides a new insight in different technique to store data into big data in the field of biometrics recognition. Big data provides high volume, high velocity and high variety of information. Big data provides the cost effective framework to store data. The scale of the biometric data to ensuring the integrity of biometric data by elimination duplicate records. 
Today, the primary application of biometrics is in physical security: to control access to secure locations (rooms or buildings). Biometric system which rely on the evidence of multiple sources of information for establishing identity are called Multimodal biometric system. This paper presents an overview of multimodal biometrics data handling through big data. It also discuss their applications to develop the security system for high security areas.

A lot of research work is still need in this area. In near future multi model biometric data handling can apply to enhance the security, real time response and cost cutting approach of our system.

\section{References:}

Research Paper

1. Taruna Panchal, Dr. AjitSingh, s "Multimodal Biometric System", International Journal of Advanced Research in Computer Science and Software Engineering, Volume 3, Issue 5, May 2013.

2. Sukhdeep Singh, Dr. Sunil Kumar Singla , "A Review on Biometrics and Ear Recognition Techniques" , International Journal of Advanced Research in Computer Science and Software Engineering, Volume 3, Issue 6, June 2013.

3. Khalid Adam Ismail Hammad, Mohammed Adam Ibrahim Fakharaldien, Jasni Mohamed Zain, Mazlina Abdul Majid, "Big Data Analysis and Storage" , Proceedings of the 2015 International Conference on Operations Excellence and Service Engineering Orlando, September 10-11, 2015.

4. Amir Gandomi, MurtazaHaider, "Beyond the hype: Big data concepts, methods, and analytics", International Journal of Information ManagementElsevier (2015), Pg no. 137-144.

5. Stephen J. Elliott, Kevin O'Connor, Eric Bartlow, Joshua J. Robertson and Richard M. Guest "Expanding the Human-Biometric Sensor Interaction Model to Identity Claim Scenarios", Research gate - Conference Paper - March 2015.

6. Elena Geanina ULARU, Florina Camelia PUICAN, Anca APOSTU, Manole VELICANU, "Perspectives on Big Data and Big Data Analytics", Database Systems Journal vol. III, no. 4/2012.

7. Ravi Subban and Dattatreya P. Mankame, "A Study of Biometric Approach Using Fingerprint Recognition" Lecture Notes on Software Engineering, Vol. 1, No. 2, May 2013.
8. ShafagatMahmudova, "Big Data Challenges in Biometric Technology", I. J. Education and Management Engineering, 2016, 5, 15-23.

9. Ms. Priya N. Ghotkar1, Prof. Vikas G. Bhowate2,

10. Julius N. Obidinnu, Ayei E. Ibor, S. O. O. Duke, "Improving the Security of MANETs Oriented Military Intelligence using Biometrics Authentication Technologies", Scientific Research Journal (SCIRJ), Volume 2, Issue 1, January 2014.

11. Prof. Vijay M. Mane, Prof. (Dr.) Dattatray V. Jadhav, "Review of Multimodal Biometrics: Applications, challenges and Research Areas", International Journal of Biometrics and Bioinformatics (IJBB), Volume 3, Issue 5.

12. Mugdhajain, ChakradharVerma," Adapting kmeans for Clustering in Big Data", International Journal of Computer Applications (0975 - 8887) Volume 101- No.1, September 2014.

13. https://www.pinkelephantasia.com/wpcontent/uploads/2017/10/Five-Vs-Big-Data.png

14. https://www.google.co.in/search?q=5+vs+of+big+ data\&dcr $=0 \&$ source $=1 n m s \&$ tbm $=$ isch $\& s a=X \& v e d$ =0ahUKEwjd8qjMvZPZAhXKuY8KHVbbBt8Q _AUICigB\&biw=1366\&bih=626\#imgrc $=$ tLYr07e oKBgB1M:

15. https://disruptionhub.com/10-uses-facialrecognition-technology/

16. https://www.dreamstime.com/s tock-illustrationeye-biometrics-retina-scan-emblem-virtualverification-identity-concept-scanning-biometrymodern-contemporary-technologyimage73611262

17. https://www.dreamstime.com/s tock-illustrationeye-biometrics-retina-scan-emblem-virtualverification-identity-concept-scanning-biometrymodern-contemporary-technologyimage 73611262

18. https://www.google.co.in/search?q=face+recogniti on $\&$ hl $=$ en $\&$ source $=$ lnms \&tbm $=$ isch $\&$ sa $=X \& v e d=$ 0ahUKEwjRwoKD5ZraAhXDLI8KHSQyCOsQ _AUICigB\&biw=1366\&bih=626\#imgrc $=w O 2 U T$ Oe9XkTsWM:

19. https://www.google.co.in/search?q=Signature+rec ognition \&source $=\operatorname{lnms} \&$ tbm $=\mathrm{isch} \& \mathrm{sa}=\mathrm{X} \& \mathrm{ved}=0$ ahUKE wiTpbCo5JraAhXLvI8KHfvMD1wQ_A UICigB\&biw=1366\&bih=675\#imgrc=boGW3Xo v5hNlYM:

20. https://www.google.co.in/?gfe_rd=cr\&ei=7xIGW YKKDPHy8AeOzYKQC A 